\title{
The Axis of Horizontal Eye Rotation of Fish*1
}

\author{
Akira Kabayama ${ }^{* 2,3}$, Gunzo KaWAmURA ${ }^{* 2}$, and Tooru Yonemori ${ }^{* 2}$ \\ (Received July 31, 1979)
}

\begin{abstract}
To clarify the axis of horizontal eye rotation of fish, a $35 \mathrm{~mm}$ camera filmed the optomotor reaction of the animal from above, and the axis of horizontal eye rotation was estimated mechanically. Moreover, the muscles and the space between the eye and the orbit were studied anatomically. The axis of the horizontal eye rotation is not a single point. The axes measured geometrically seem to lie around an optic center of an eye, and the axis tended to shift a little bit nasalward when the eye rotated nasalward.
\end{abstract}

Fishes capable of moving head and eyes usually coordinate the two reciprocally, so that as the head moves outside, the eyes slew to the counter, keeping the retinal images relatively stable ${ }^{1-4)}$. The full range of horizontal eye displacement was reported as $41.5 \pm 2.5$ degrees in goldfish ${ }^{21}, 34$ degrees in crucian carp $^{41}$. The maximum saccade velocity was reported as 0.77 degrees/ms in goldfish ${ }^{3 /}$ and 1.16 degrees/ms in crucian carp ${ }^{4}$.

These were measured mainly cinematographically, depending on the assumption that eyes may totate around a single point. HermanN and CONSTANTINE ${ }^{1 /}$ suggested that the eye of fish does not appear to rotate around a single point. If the axis is known, we might be able to make the cinematographical angular measurement of eye position more accurate. In this paper, we report on the axis of horizontal eye rotation in freshwater teleosts.

\section{Materials and Method}

A wild largemouth bass, Micropterus salmoides, $33.5 \mathrm{~cm}$ in total length, and a wild bluegill, Lepomis macrochirus, $18.5 \mathrm{~cm}$ in total length used in this study were caught in the Satsuma Lake in Kagoshima Prefecture. A crucian carp, Carassius auratus langsdorfi, $20.5 \mathrm{~cm}$ in total length, used was obtained from a local supplier. Each of them was kept in an indoor tank. Immediately prior to the experiment, following to KAWAMURA et al. ${ }^{4}$, two sticks ( $1 \mathrm{~mm}$ in width, $4 \mathrm{~mm}$ in length) were at- tached to the right cornea above the line of sight. As the eye moved, so did the sticks. To immobilize the animal, it was clamped just behind the gill flaps between two plates of towel-padded vinyl chloride. And its mouth was fitted around an inlet tube through which aerated water flowed to irrigate the gills, as schematized in Fig. 1.

For the cinematographical record of continuous eye movement, moving target was presented on a rotating belt. The subtended $30^{\circ}$ of the animal's visual field in the vertical plane and $118^{\circ}$ horizontally. Its outer surface was lined with alternating black and white stripes subtending angles of $8^{\circ}$ at the fish's eye, and moved about the vertical axis at 20-40 degrees/s nasaly or caudally. A $35 \mathrm{~mm}$ camera, Canaon F-1, with a film chamber and a motor drive unit filmed the optomotor reaction (OMR) of the animal from above. Filming rate was 3 frames/s.

If one horizontal eye rotation is done around a single point, we can geometrically regard a point of intersection of two nomal bisectors of chords of the arcs drawn by each stick as an axis of the rotation (Fig. 2). For graphic analysis, some sequences of frames containing the clear OMR were selected. and each frame was printed into enlarged photographs. The trace of the eye rotation was obtained by superimposing the positions of the sticks.

After the filming, the animal was killed, and the eye and orbit were observed anatomically.

Prior to this experiment, the retina of other animals of the same species were observed histologi-

*1 This was presented at the annual meeting of the Japanese Society of Scientific Fisheries on October 1978, Shimizu.

*2 Laboratory of Fishing Technology, Faculty of Fisheries, Kagoshima University, Kagoshima 890, Japan (松山 明 - 川村軍藏・米盛 字：倠胃島大学水産学部澹法学教室).

*3 Present address: Nippon Oceanic Survey Co. Ltd., Minato 7-8, Moji, Kitakyushu 801, Japan (日本海 洋测量株式会社). 


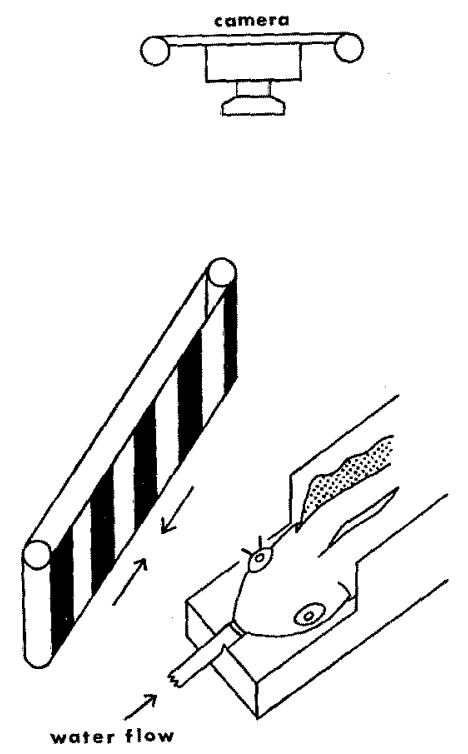

Fig. 1. Schematic presentation of the method for recording optomotor reaction.

cally, and, following TAMURA, ${ }^{5 ?}$ the minimum separable angle for each fish was calculated.

The scoring errors of the axis position lay within the limits of $0.2 \mathrm{~mm}$ in diameter for counterclockwise rotation and $0.4 \mathrm{~mm}$ in diameter for clockwise rotation.

\section{Results and Discussion}

Minimum separable angle measured histologically was 19.2 minutes in crucian carp, 10.8 minutes in largemouth bass, and 19.8 minutes in bluegill. Hence, it can be said that the animals could discriminate the black and white stripes and the angle

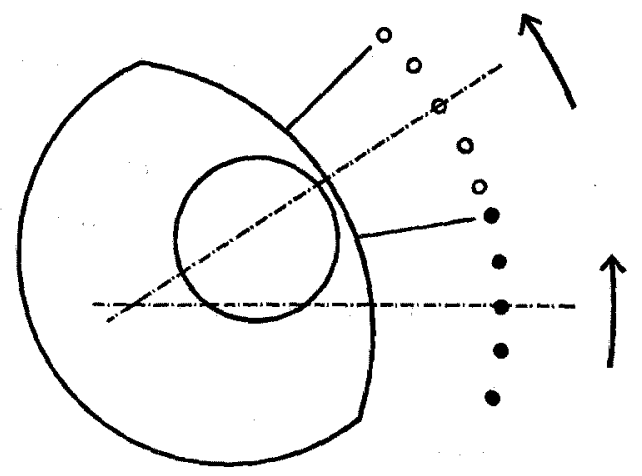

Fig. 2. Geometrical determination of the axis of the horizontal eye rotation (see also text).

subtended by the stripes was enough to evoke OMR. As the target moved, so did the eye.

The axes in three species were obtained by the same treatment when an eye rotated counterclockwise or clockwise, as shown in Fig. 3.

The axes measured geometrically seem to lie around an optic center* of an eye. When the right eye rotates counterclockwise, the axis tends to shift a little bit nasalward. Though the range of this shift is small, the scoring error does not affect so much as to deny this tendency. Thus, it can be concluded that the position of the axis obtained here are somewhat reliable and the axis of the horizontal eye rotation is not a single point.

In Fig. 4A, the extra-ocular muscles which may have an important role in eye rotation were shown schematically. An eye ball rotates in an orbit owing to the contraction of the extra-ocular muscles. In birds and snakes, the eye can not be moved because of their eyes filling the orbit, so the ocular movements are taken the place of the head

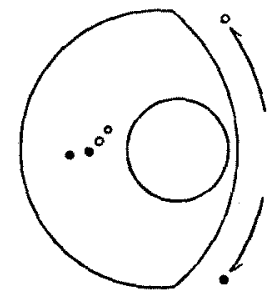

Largemouth bass

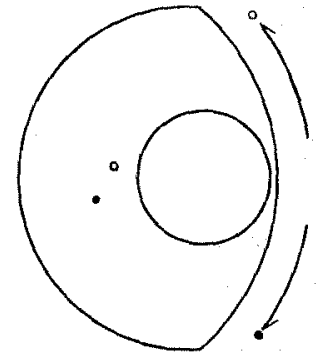

Crucian carp

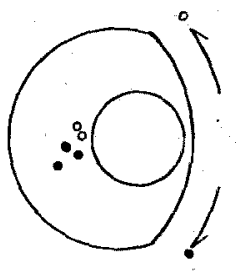

Bluegill

Fig. 3. The positions of axes determined geometrically in counterclockwise (open circle) and clockwise (closed circle) rotations of the right eye for three species.

* Middle point between the center of lens and the retinal bottom region. 

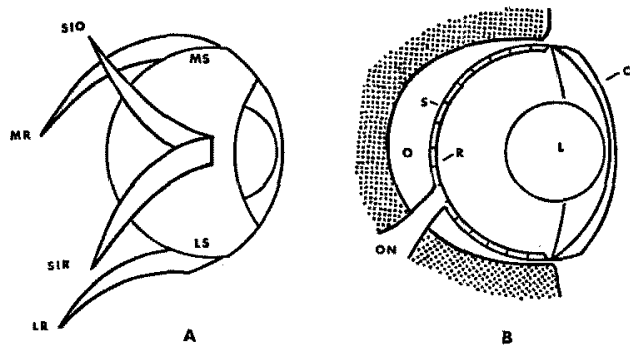

Fig. 4. Schema showing dorsal view of the arrangement of the extra-ocular muscle (A) and anatomical structure of eye and orbit (B). LR, lateral rectus; MR medial rectus; SIO, superior oblique: SIR, superior rectus; (inferior oblique and inferior rectus are not shown); MS and LS, attached points of MR and LR with the oculus; $C$, cornea; $L$, lens; $O$, orbit; On, optic nerve; $R$, retina; $S$, sclera.

movements as nodding, swivelling or swinging movements ${ }^{6 !}$. On the other hand, the space between the eye and the orbit in fish is wide, especially at the bottom (Fig. 4B). The tissue which lie in the space may fill the role of the lubricant. Then the eyes of fish can be moved well.

The fact that an eye does not rotate around a single point is not surprising, and we can not find out any rotating mechanism without a fixed axis. Though the shift of the axis between clockwise and counterclockwise rotations probably depends only on the arrangement of the extra-ocular muscles, the shift may have some advantage in eye rotation. According to the principles of the lever, the active point transfers longer distance when a fulcrum is closer to the dynamic point position. This mechanical idea can be applied to the eye rotation. For example, regarding the attaching points of $\mathrm{MS}$ and LS with an eye, and the axis respectively as the dynamic point, the active point, and fulcrum point in counterclockwise rotation, the fulcrum point is closer to the dynamic point than the active point (abbreviations as in Fig. 4). Thus by a smaller contraction of the medial rectus, the eyes can be rotated well. The very quick eye movements may partly depends on this mechanism.

We could estimate the position of the axes of the horizontal eye rotation, but could not do it so accurately as to modify the cinematographical treatment. Therefore, in cinematographical angular measurement of eye positions, we must depend on the assumption that eye rotates around a single point at this stage.

\section{References}

1) H.T. Hermann and M.M. Constantine: Vision Res., 11, 313-331 (1971).

2) S. S. EAster Jr., P. R. Johns, and D. HeCkenLIVELY: J. Comp. Physiol, 92, 23-35 (1974).

3) S.S. EASTER Jr. and P.R. JohNS: J. comp. Physiol., 92, 37-57 (1974).

4) G. Kawamura, A. Kabayama, and T. Yonemori: Bull. Japan. Soc. Sci. Fish., 44, 567-570 (1978).

5) T. TAmUra: Bull. Japan. Soc. Sci. Fish., 22, 536557 (1957).

6) S. Dukz-Elder: System of Ophthalmology, Henry Kimpton, London, 1958, pp. 273-331 and 666-707. 